\title{
Fluorination as effective method for tuning of the electromagnetic re- sponse of graphene
}

\author{
N. Valynets ${ }^{1}$, A. Paddubskaya ${ }^{1}$, P. Kuzhir ${ }^{* 1,2}$, V. Sysoev ${ }^{3}$, L. Bulusheva ${ }^{3}$, and A. Okotrub \\ ${ }^{1}$ Institute of Nuclear Problems of Belarusian State University, Bobruiskaya Str. 11, 220030 Minsk, Belarus \\ 2 Tomsk State University, 634050 Tomsk, Russia \\ ${ }^{3}$ Nikolaev Institute of Inorganic Chemistry, SB RAS, 3Acad. Lavrentiev Ave., 630090 Novosibirsk, Russia
}

Received ZZZ, revised ZZZ, accepted ZZZ

Published online ZZZ(Dates will be provided by the publisher.)

Keywords Fluorographene, thin films, microwaves, terahertz, electromagnetic shielding

*Corresponding author: e-mail polina.kuzhir@gmail.com,Phone: +375 172007410

\begin{abstract}
Fluorographene (FG) has been obtained by thermal expansion of fluorinated graphite with a $\sim \mathrm{C}_{2} \mathrm{~F}$ composition at $800^{\circ} \mathrm{C}$. Comparative study of electromagnetic (EM) response of thin fluorographene films after recovering their conductivity via different methods is presented in
\end{abstract}

microwave $(\mathrm{GHz})$ and terahertz $(\mathrm{THz})$ frequency ranges. We discovered that fluorination can be used as promising tool for producing tunable EM interference (EMI) shielding materials, combining reflective and absorptive layers.

Copyright line will be provided by the publisher
1 Introduction Graphene is carbon two-dimensional honeycomb crystal lattice demonstrating outstanding optic, electromagnetic and mechanical properties. Being "pristine", graphene is zero band gap semiconductor, which makes difficult to use it for $\mathrm{AC}$ applications, i.e. for the realization of graphene-based high-frequency ballistic devices as well as the design of graphene-based integrated circuits capable of fully utilizing its unique electronic properties. One of the simple ways to influence the electronic "fingerprints" of graphene, and thereby its electromagnetics, is chemical modification via oxidation [1], hydrogenation [2], and fluorination [3]. Next thermal decomposition of functional groups and intercalated molecules creates the pressure, which overcomes the Van der Waals attractions between the layers [4].

Recently, we demonstrated that nano-thin conductive carbon and graphene films could be highly absorptive at certain thickness which is inversely proportional to its conductivity [5-10]. The idea of this communication is to present a tunable EMI shidding layer based on fluorog. raphene

Due to the possibility to open and more importantly to vary the band gap in graphene with covalent modification by means of fluorine, currently fluorographene (FG) attracts huge interest for many practical applications. It is known that fluorographene is dielectric material with bandgap energy of $3 \mathrm{eV}$ [11]. There are various methods for preparing fluorographene such as treatment of the material in a gaseous atmosphere $\left(\mathrm{F}_{2}, \mathrm{XeF}_{2}, \mathrm{BrF}_{3}, \mathrm{ClF}_{3}\right)$ [12] or plasma, fluorination at room temperature and atmospheric pressure in an aqueous solution of hydrofluoric acid (HF) [13].

In present work, it is demonstrated that the fluorinated graphene obtained by thermal expansion of a bromineintercalated fluorinated graphite compound, as its reduction (recovering of the conductivity through the simple methods, i.e. chemical reduction in hydrazine-hydrate vapour and heat treatment in vacuum) leads to the changing of AC conductivity level and thereby to changing its EMI shielding ability.

\section{Experimental details}

2.1 Materials Materials Natural graphite from Zavalievo deposit (Ukraine) with the particles sizes about 400 $\mathrm{m}$ and thickness $20 \mathrm{~m}$ was used as a starting material. Initial few-layered graphene was prepared by rapid exfoliation of natural graphite fluoride in a quartz reactor at $800{ }^{\circ} \mathrm{C}$ in an ambient atmosphere. The temperatures were chosen based on the thermal decomposition data for such kind of fluorinated graphite compounds [14].

The fluorinated graphene was synthesized by gasphase fluorination of few-layered graphene with the soluti- 
on of $\mathrm{BrF}_{3}$ at $\mathrm{Br}_{2}$ at room temperature as described elsewhere [15]. On the first stage, few-layered graphene was put in a teflon flask with perforated wall and placed in a hermetic reactor containing solution of $\mathrm{BrF}_{3}$ in $\mathrm{Br}_{2}$. The flask content was dried in a nitrogen flow to remove the non-reacted species. As a result of the fluorination procedure is an intercalation compound where $\mathrm{Br}_{2}$ molecules are included between fluorinated graphene layers of approximate $\mathrm{C}_{2} \mathrm{~F}$ composition. Detailed characterization of the compound can be found elsewhere [16].

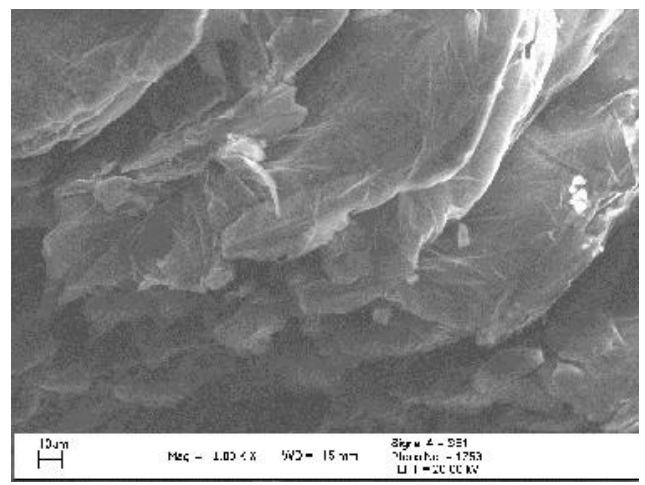

Figure 1 SEM images of fluorographene particles.

Scanning electron microscopy (SEM) with a LEO1455 VP microscope was used for structural characterization of material. The SEM images of obtained FG particles are presented in Fig. 1.

2.2 Fluorographene films preparation and characterization First, the solution containing $1 \mathrm{mg} / 5 \mathrm{ml}$ of fluorographene powder was prepared in a volume of 20 $\mathrm{ml}$ an organic solvent toluene at $20^{\circ} \mathrm{C}$. This resulting solution was submitted under ultrasonication using a bath sonication at $100 \mathrm{~W}$ for 1 hour. The color of a fluorographene suspension changes during ultrasonic treatment with a brown to a light black.

The thin FG films have been produced by spin-coating. The prepared solution was dropped onto the quartz substrate, which was rotated at $5000 \mathrm{rpm}$ for $750 \mathrm{~s}$ and $2250 \mathrm{~s}$. In order to measure the EM properties in microwave frequency range using the very simple technique, the size of the quartz substrate was $7.2 \times 3.4 \times 0.5 \mathrm{~mm}$. The fluorinated suspension easily spread over the surface of the substrate to form a thin continuous film (Fig. 2).

There are several ways of recovering the conductivity of the modified on-conducting CF films such as irradiation by electron beam [17], heat treatment in a vacuum and by the action of hydrazine-hydrate vapour [18]. In present work, in order to change the fluorine concentration the following two methods were used:

1. For chemical reduction of the sample surface is a direct treatment of FG films with $1 \%$ hydrazine-hydrate vapour during $10 \mathrm{~min}$ as one of the simplest.
2. The second method is heat treatment in a vacuum. As known from the literature, $\mathrm{CF}$ is stable in vacuum up to $400{ }^{\circ} \mathrm{C}$ [19]. Therefore FG samples on a dielectric substrate were heated in a vacuum $\left(10^{-6}\right.$ Torr $)$ at $550{ }^{\circ} \mathrm{C}$ for $10 \mathrm{~min}$ for changing the level of fluorine and evaporating the residue of the solvent and the possible organic impurities.

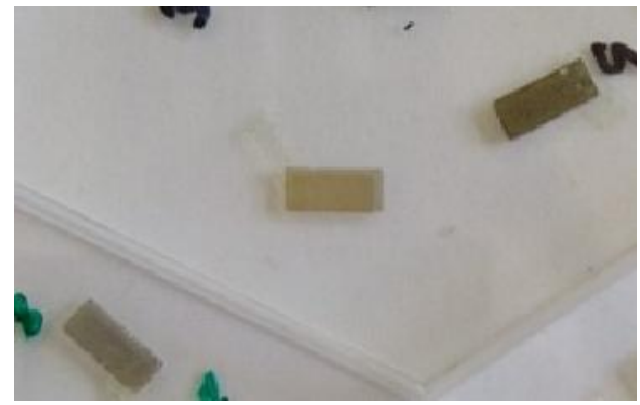

a)

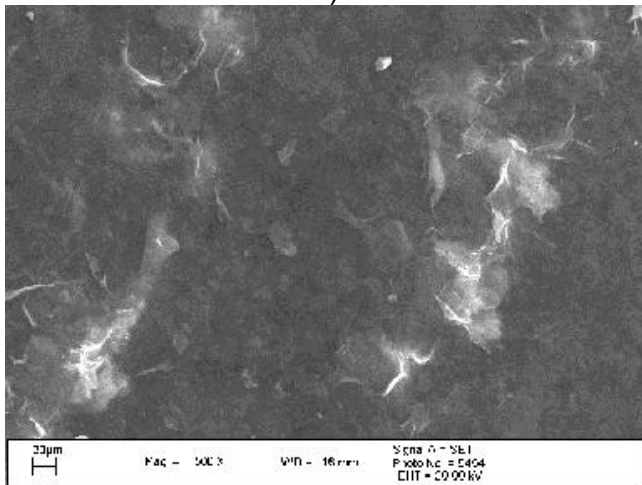

b)

Figure 2 Photo image of FG thin film on a quartz substrate (a). SEM image of film's surface (b).

In order to investigate the influence of fluorine concentration and film thickness to the resulting EM response of carbon films, the samples with two different thicknesses have been produced (denote as "thin" and "thick"). The film's thickness was measured by a stylus profiler (Veeco Instruments, Dektak 150, Tucson, AZ, USA) with an accuracy of $1.5 \mathrm{~nm}$. The thickness was averaged over ten different points with the variation $\pm 3 \mathrm{~nm}$ (see Table 1).

Table 1 The presence of fluorine (wt. \%) in FG films, and their AC conductivity $(30 \mathrm{GHz})^{1}$

\begin{tabular}{|c|c|c|c|}
\hline $\begin{array}{c}\text { Thickness, } \\
\text { nm }\end{array}$ & $\begin{array}{cc}\text { F wt., } \%, & \text { F wt., } \%, \\
\text { before } & \text { after } \\
\text { treatment annealing }\end{array}$ & $\begin{array}{cc}\text { F wt., } \%, & \sigma_{\mathrm{AC}} 10^{3} \\
\text { after } & \mathrm{S} / \mathrm{m}, \text { af- } \\
\text { treatment } & \text { ter } \\
\text { with } \mathrm{HH} \text { annealing }\end{array}$ & $\begin{array}{l}\sigma_{\mathrm{AC}} 10^{3} \\
\mathrm{~S} / \mathrm{m}, \text { af- } \\
\text { ter } \\
\text { treatment } \\
\text { with HH }\end{array}$ \\
\hline 50 & 0.55 & 40.05 & 44.40 \\
\hline 120 & 1.11 & 89.01 & 28.93 \\
\hline
\end{tabular}

${ }^{1}$ No significant frequency dependence in wide frequency range was observed $(30 \mathrm{GHz}-1 \mathrm{THz})$, which is inherent to all graphene-like samples (see e.g. [10]). 
To control the level of fluorine after each treatment the element analysis of films was carried out (see Table 1). As it follows from EDX analysis there are 1.82 wt. \% and 9.71 wt. \% of fluorine in initial "thin" and "thick" films, respectively. In contrast, the content of fluorine after annealing dramatically decrease up to 0.55 wt. $\%$ and 1.11 wt. $\%$, respectively.

Raman scattering was further used to analyze the quality of graphene layers. Measurements were performed by Raman spectrometer combined with a confocal Raman spectrometer Nanofinder HE (LOTIS TII, Belarus-Japan) with a $600-$ lines $/ \mathrm{mm}$ grating and $532-\mathrm{nm}$ laser excitation. It is well known that three main futures usually observe in graphite-based materials: $D$ and $G$ bands located at $\sim 1341 \mathrm{~cm}^{-1}$ and $\sim 1585 \mathrm{~cm}^{-1}$, respectively, and 2D band located at $\sim 2680 \mathrm{~cm}^{-1}$. The Raman spectra of investigated samples after normalizing to the intensity of $\mathrm{G}$ band $\left(\sim 1585 \mathrm{~cm}^{-1}\right)$ are presented in Fig. 3. Note, as it was demonstrated in Ref. [20], additionally, the weak shoulder $\mathrm{D}^{\prime}\left(1620 \mathrm{~cm}^{-1}\right)$ band is usually observed in Raman spectra of fluorographene. This band indicates of a coexistence of conjugative carbon-carbon bonds and $\mathrm{sp}^{3}$-hybridized carbon atoms covalently bonded with fluorine. As it can be seen, significant D band, associated with disorder-induced symmetry-lowering effects, is typical for fluorographene. Let us note that not only defect regions contributed to the intensity of D band, its value also dependents on the flake size.

The relevant quality of graphitic materials can be estimated using the intensity ratio of D band $\left(I_{D}\right)$ and $\mathrm{G}$ band $\left(I_{G}\right)$. In our case, before treatment, the ratio $I_{D} / I_{G}$ is 0.98 and 1.02 for "thick" and "thin" samples, respectively, which mean a decrease in the average size of the $\mathrm{sp}_{2}$ domains upon reduction of fluorographene. As it was found the relative intensity $I_{D} / I_{G}$ increases up to 1.03 and 1.57 for "thick" and "thin" samples exposed to hydrazine hydrate vapor. Note, in the case of "thin" film after annealing and hydrazine-hydrate treatment the intensity second-order 2D peak also increase indicating the evolution of the structural and electronic properties of our samples.

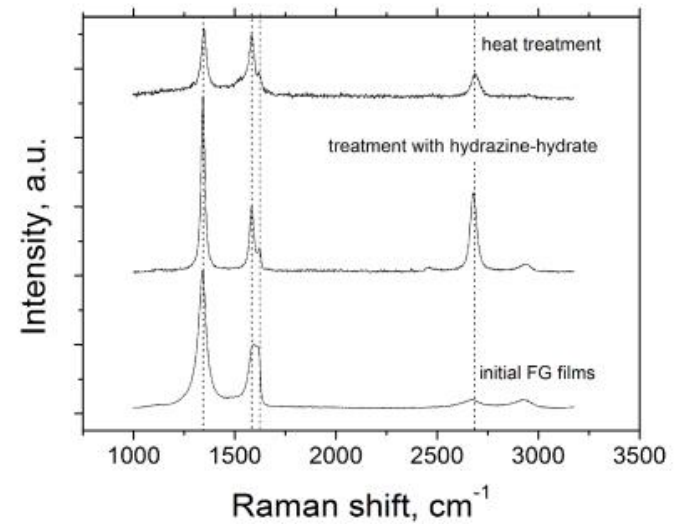

a)

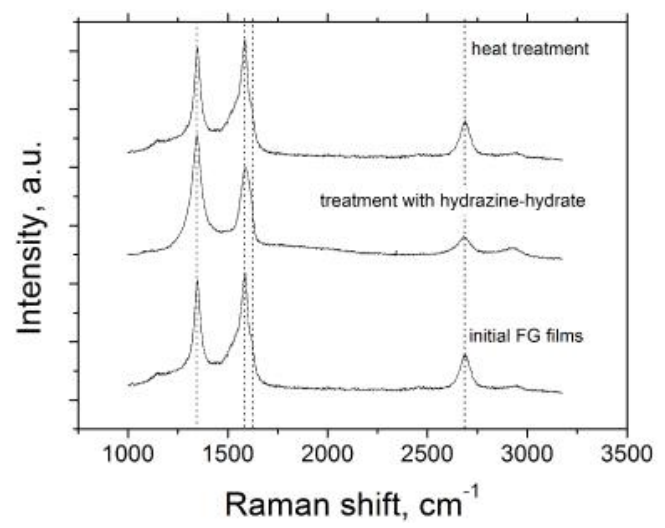

b)

Figure 3 Raman spectra before and after treatment: (a) "thin" FG films, (b) "thick" FG films. All spectra were normalized on the intensity of G band. Fluorescent background was subtracted from the spectra.

2.3 Electromagnetic characterization The microwave measurements were carried out by scalar network analyzer R2-408R (ELMIKA, Vilnius, Lithuania) in 26-37 $\mathrm{GHz}$ frequency range ( $\mathrm{Ka}$-band). The EM response of investigated samples was measured as ratio of transmitted/input $\left(S_{21}\right)$ and reflected/input $\left(S_{11}\right)$ signals. The experimental setup is described in details in previous work [5]. Reflection $(R)$, transmission $(T)$, and absorption $(A)$ are calculated from the measured $S$-parameters as $R=S_{11}^{2}$, $T=S_{21}^{2}, A=1-T-R$. The THz transmission spectra are collected with THz time-domain spectrometer T-SPEC Ekspla (Lithuania). The measurements details can be found in [9].

3 Results and discussion As it follows from our undertaken study the frequency-dependence of measured $S$ - parameters for FG films doesn't show any features, for example, absorption peak or strong frequency dependence within the selected frequency range, therefore all results are presented at one frequency $(30 \mathrm{GHz})$.

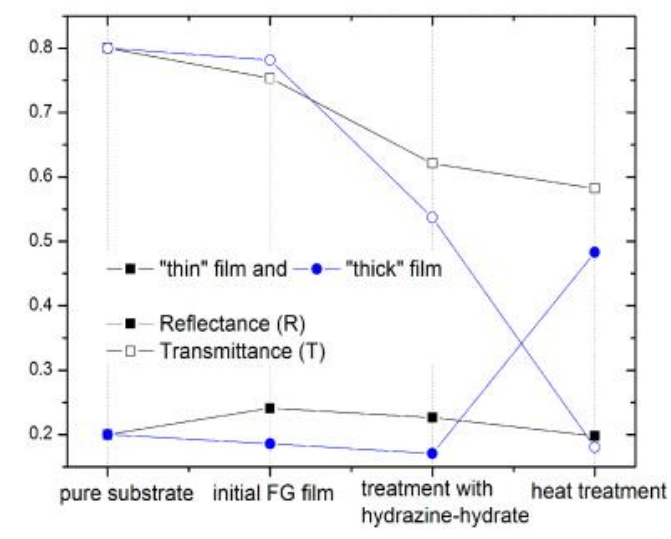

a) 


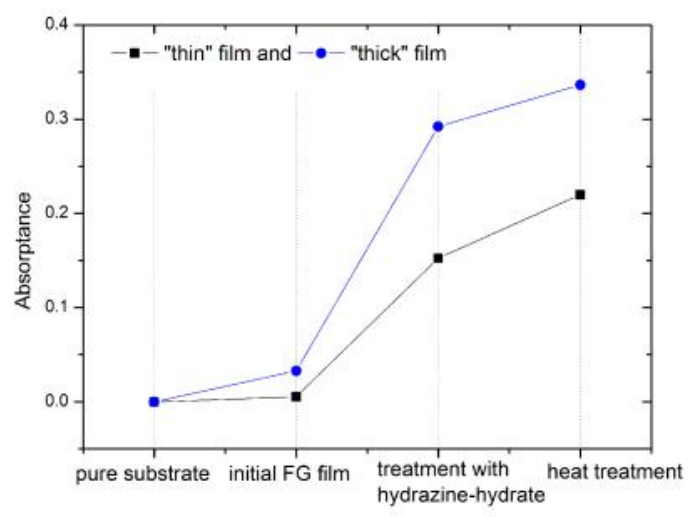

b)

Figure 4 Reflectance $(R)$, transmittance $(T)$ (a) and absorptance (A) (b) of fluorographene films at $30 \mathrm{GHz}$ for "thin" and "thick" films after annealing in vacuum and treatment with hydrazinehydrate vapor.

As one can see from Fig. 4, the FG films before treatment are almost transparent for microwave radiation $(T$ $75 \%$ and $T \sim 78 \%$ for films with thickness $50 \mathrm{~nm}$ and 120 $\mathrm{nm})$, whereas after treatment with hydrazine-hydrate vapour the transmission coefficient $(T)$ decreases to the level $62 \%$ and $54 \%$ for "thin" and "thick" samples, respectively. Such behaviour can be explained by an increase of the absorption ability $(A)$ of such structures. In particular, the absorptance of "thick" films increases up to $30 \%$ after treatment with hydrazine hydrate and up to $35 \%$ after annealing. In contrast, after $10 \mathrm{~min}$ annealing in vacuum the transparency decreases up to $59 \%$ and $18 \%$ for films with thickness $50 \mathrm{~nm}$ and $120 \mathrm{~nm}$, respectively. The main contribution to the EM attenuation comes from reflection. Moreover, as it can be observed the reflectance ability of samples higher $(R \sim 48 \%)$ in the case of sample with larger thickness. At the same time, the absorption of EM radiation is also significant $(22 \%$ and $34 \%$ for "thin" and "thick" films, respectively). This means that after some annealing time a transition to the conductive state is observed, indicating a change in the fluorination degree of samples (decrease it), which means too that graphitic regions with different defects are formed after annealing because of removal of fluorine atoms. It is know from classical electromagnetics that for every conductive material there exist the optimal thickness, which is inversely proportional to its conductivity for which one may achieve $50 \%$ of absorption. The larger the conductivity, the smaller thickness will be necessary for $50 \%$ absorption. For instance for CVD graphene of average quality we need 4-7 layers [8,9], that is $10-15 \mathrm{~nm}$; for graphite it is $100 \mathrm{~nm}$ [6], for pyrolytic and glassy carbon it is $75 \mathrm{~nm}[6,10]$. As for $120 \mathrm{~nm}$ film of fluorinated graphene we reach $34 \%$ of absorption, it means that optimal thickness is approx. $160 \mathrm{~nm}$, and conductivity of annealed FG is slightly smaller than conductivity of graphite.

The transmittance of FG films on quartz substrate in $\mathrm{THz}$ frequency range is presented in Fig. 5. For compari- son, the transmittance spectrum of pure quartz is presented too (dot curve). It is important to note that the observed frequency dispersion is due to the effect of interference on a quartz substrate and small shift of interference peaks is related to the difference in thickness of quartz substrate. As it follows from Fig, 5, initial FG film is transparent for $\mathrm{THz}$ radiations $(\sim 80 \%)$ that indicate the dielectric properties of such kind materials. At the same time, the annealing of "thick" FG film leads to strong reduction of the transmittance level from $80 \%$ up to $10 \%$. For samples after treatment with hydrazine-hydrate vapour the transmission coefficient is reduced up to $40 \%$. It should be noted that the effect of hydrazine-hydrate treatment does not dependent on the film thickness which indicates that only surface layer of films is reduced in this way. This conclusion is in agreement with EDX results (see Table 1).

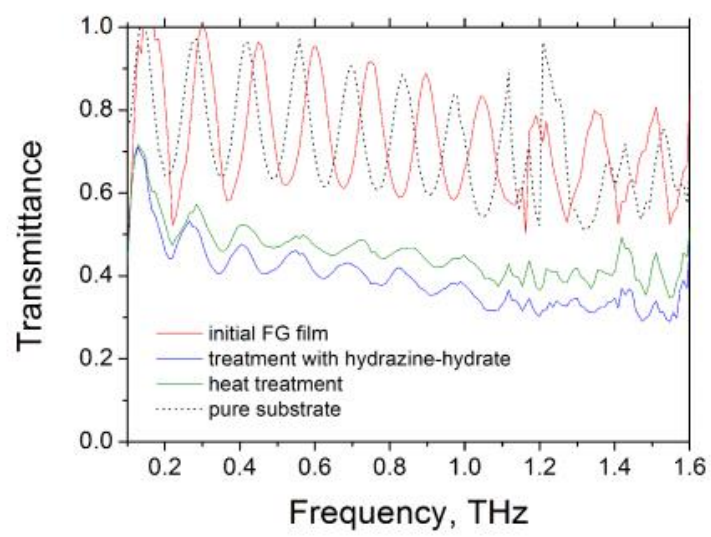

a)

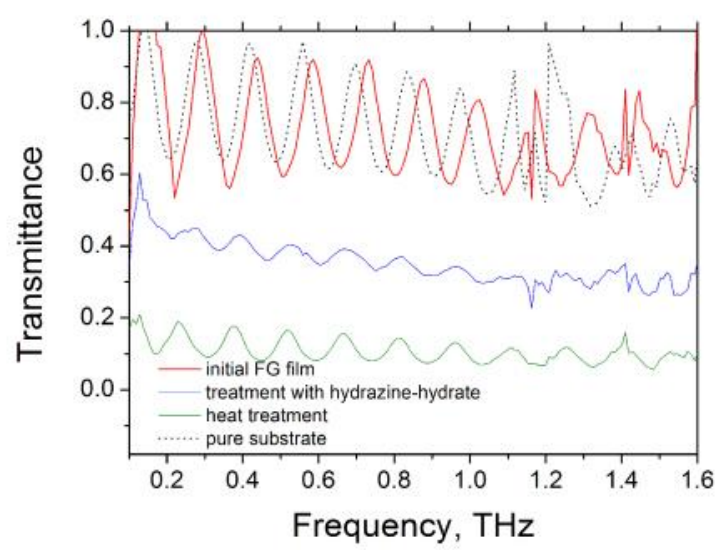

b)

Figure 5 Terahertz transmittance spectra of "thin" (a) and "thick" (b) FG films before and after treatment.

The effective thin films conductivity (see Table 1) was extracted from the experimental data using the procedure detailed elsewhere $[6,21]$. In the numerical calculation, the dielectric permittivity of pure quartz was set $3.8+0.025 \mathrm{i}$. Note, as it was mention above, the initial FG films are transparent for microwave and $\mathrm{THz}$ radiation which, in 
turn, results in the impossibility to extract the dielectric properties of such kind films, having in mind its invisible thickness in comparison with the wavelength $(300 \mathrm{mi}-$ crons), as for conductive film of the same thickness the absorption would be significant (se e.g. [10] for the task statement details).

From comparison of the calculated results and film's elemental analysis the following conclusion can be made:

- Annealing of FG leads to more effective reduction of such kind material and development of conductive surface layer.

- Hydrazine-hydrate can be used as a reductant, but the effectiveness of such treatment depends on the film thickness. In particular, the conductivity of "thin" film is the same after annealing and hydrazine-hydrate treatment. In contrast, in case "thick" films the conductivity of samples after annealing is three times more.

\section{Conclusions}

We demonstrated that using various methods of recovering the conductivity of thin fluorographene films by regenerating graphene from graphene fluoride such films can provide different levels of absorption / reflection of EM signal. These characteristics can be easily changed by varying the films thickness or treatments duration, providing a way to tune the electromagnetic characteristics from microwave up to $\mathrm{THz}$ frequency ranges. Summarizing, we can conclude that along with graphene nanoplatelets containing polymer composites (see e.g. [21,22]), such thin films can be very suitable materials for producing effective absorbers, electromagnetic shielders, design of highfrequency power detectors and sensors for microwave and $\mathrm{THz}$ radiations.

Acknowledgements This work was carried out within the framework of the H2020 project 696656 Graphene Core1, H2020 project $644076 \mathrm{CoExAN}$, and RFBR, according to the research project No.15-33-50490 «mol_nr», U.S. Air Force through CRDF Global Agreement grant AF20-15-618041. PK is thankful for support by Tomsk State University Competitiveness Improvement Program.

\section{References}

[1] D. R. Dreyer, S. Park, C. W. Bielawski, R. S. Ruoff, Chem. Soc. Rev 39, 228 (2010).

[2] D. C. Elias, R. R. Nair et al., Science 323, 610 (2009).

[3] R. R. Nair, et al., Small 6, 2877 (2010).

[4] M. Cai, D. Thorpe, D. H. Adamson, and H. C. Schniepp, J. Mater. Chem. 22, 24992 (2012).

[5] P. Kuzhir, A. Paddubskaya, S. Maksimenko, T. Kaplas nd Yu. Svirko, Nanoscale Research Letters, DOI 10.1186/1556276X-8-60 (2013).

[6] K. Batrakov, P. Kuzhir, S. Maksimenko, A. Paddubskaya, S. Voronovich, T. Kaplas, and Yu. Svirko, Applied Physics Letters 103, 073117 (2013).
[7] P. Kuzhir, N. Volynets, S. Maksimenko, T. Kaplas and Yu. Svirko, J. Nanosci and Nanotechn 13, 5864 (2013).

[8] K. Batrakov, et al, Scientific Reports 4, 7191 (2014).

[9] K. Batrakov, et al, Appl.Phys. Lett. 108, 123101 (2016).

[10] P. Kuzhir, A. Paddubskaya, N. Volynets, K. Batrakov, T. Kaplas, P. Lamberti, R. Kotsilkova, Ph. Lambin, J. Nanophoton. 11, 032504 (2017).

[11] R.R. Nair, W. Ren, R. Jalil, I. Riaz, V.G. Kravets, L. Britnell, P. Blake, F. Schedin, A.S. Mayorov, S. Yuan, M.I. Katsnelson, H.-M. Cheng, W. Strupinski, L.G. Bulusheva, A.V. Okotrub, I.V. Grigorieva, A.N. Grigorenko, K.S. Novoselov, A.K. Geim, Small 6, (2010) 2877-2884.

[12] N. A. Nebogatikova, I. V. Antonova, V. Ya. Prinz, et al., Phys.Chem.Chem.Phys. 17, 13257 (2015).

[13] N. F. Ydanov, A. V. Okotrub, et al., Journal of Inorganic Chemistry 45, 1960 (2000).

[14] Yu. V. Fedoseeva, A. V. Okotrub, I. P. Asanov, D. V. Pinakov, G. N. Chekhova, V. A. Tur, P. E. Plyusnin, D. V. Vyalikh, and L. G. Bulusheva, Phys. Status Solidi B 251, 2530 (2014).

[15] I. P. Asanov, et al., Carbon 57, 518 (2013).

[16] A. V. Okotrub, N. F. Yudanov, I. P. Asanov, D. V. Vyalikh, and L. G. Bulusheva, ACS Nano 7, 65 (2013).

[17] I. P. Asanov, A. V. Okotrub, A. V. Gusel'nikov, I. V. Yushina, D. V. Vyalikh, L. G. Bulusheva, Carbon 82, 446 (2015).

[18] A. V. Okotrub, K. S. Babin, A. V. Gusel'nikov, I. P. Asanov and L. G. Bulusheva, Phys. Status Solidi B 247, 3039 (2010).

[19] S.-H. Cheng, . Zou, F. Okino, H.R. Gutierrez, A. Gupta, N. Shen, P.C. Eklund, J.O. Sofo, J. Zhu, J. Phys. Rev. . 81, 205435 (2010).

[20] V. Gupta, T. Nakajima, Y. Ohzawa, and B. Žemva, J. Fluor. Chem. 120, 143 (2003).

[21] A. Paddubskaya, N. Valynets, P. Kuzhir, K. Batrakov, S. Maksimenko, R. Kotsilkova, H. Velichkova, I. Petrova, I. Biro, K. Kertesz, G.I. Mark, Z.E. Horvath, L.P. Biro, J. Appl. Phys. 119, 135102 (2016).

[22] P. Kuzhir, A. Paddubskaya, A. Plyushch, et al, Journal of Applied Physics 114, 164304 (2013). [24] I. Kranauskaite, J. Macutkevic, P. Kuzhir, et al, Physica

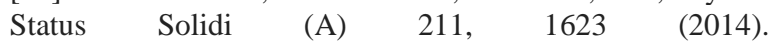

